\title{
The MAP3K13-TRIM25-FBXW7a axis affects c-Myc protein stability and tumor development
}

\author{
Qiang Zhang ${ }^{1,2} \cdot \mathrm{Xu} \mathrm{Li}^{1,2} \cdot \mathrm{Kasa}^{\mathrm{Cui}}{ }^{1,2} \cdot$ Cheng $\mathrm{Liu}^{1,2} \cdot$ Mingzhi Wu${ }^{1,2} \cdot$ Edward V. Prochownik $^{3} \cdot$ Youjun $\mathrm{Li}^{1,2}$
}

Received: 28 December 2018 / Revised: 17 May 2019 / Accepted: 28 May 2019 / Published online: 11 June 2019

(c) The Author(s), under exclusive licence to ADMC Associazione Differenziamento e Morte Cellulare 2019

\begin{abstract}
c-Myc (Myc) is a master transcription factor that is often deregulated and highly expressed by at least $50 \%$ of cancers. In many cases, Myc protein levels correlate with resistance to therapy and poor prognosis. However, effective direct inhibition of Myc by pharmacologic approaches has remained unachievable. Here, we identify MAP3K13 as a positive regulator of Myc to promote tumor development. Our findings show that MAP3K13 upregulation is predictive of poor outcomes in patients with hepatocellular carcinoma (HCC). Mechanistically, MAP3K13 phosphorylates the E3 ubiquitin ligase TRIM25 at $\operatorname{Ser}_{12}$ to decrease its polyubiquitination and proteasomal degradation. This newly stabilized TRIM25 then directly ubiquitinates Lys $_{412}$ of FBXW7 $\alpha$, a core subunit of the SKP1-Cullin-F-box (SCF) ubiquitin ligase complex involved in Myc ubiquitination, thereby stabilizing Myc. Together, these results reveal a novel regulatory pathway that supervises Myc protein stability via the MAP3K13-TRIM25-FBXW7 $\alpha$ signaling axis. In addition, they provide a potential therapeutic target in Myc over-expressing human cancers.
\end{abstract}

\section{Introduction}

Hepatocellular carcinoma (HCC) is the fifth most prevalent cancer worldwide and accounts for $\sim 745,500$ death per year $[1,2]$. The incidence of HCC has tripled in the last two decades, but the survival rate remains exceedingly low [3]. Most HCC patients are diagnosed at an advanced stage, when surgical resection, transplantation, and percutaneous

Edited by D. Guardavaccaro

Supplementary information The online version of this article (https:// doi.org/10.1038/s41418-019-0363-0) contains supplementary material, which is available to authorized users.

Youjun Li

liy7@whu.edu.cn

1 Hubei Key Laboratory of Cell Homeostasis, College of Life Sciences, Wuhan University, 430072 Wuhan, China

2 Medical Research Institute, School of Medicine, Wuhan University, 430071 Wuhan, China

3 Division of Hematology/Oncology, Children's Hospital of Pittsburgh of UPMC, The Department of Microbiology and Molecular Genetics and The Hillman Cancer Center of UPMC, The University of Pittsburgh Medical Center, Pittsburgh, PA 15224, USA ablation are no longer feasible [4, 5]. Therefore, new effective treatments for improving survival will require novel insights into the precise molecular mechanisms underlying the basic mechanisms that support HCC growth.

$\mathrm{HCC}$ is a heterogenous disease driven by the serial accumulation of mutations in tumor suppressor genes and proto-oncogenes [6]. c-Myc (Myc) is one of the most commonly activated oncogenes in HCC. Previous studies have revealed that Myc overexpression is sufficient to induce liver cancer in animal models, whereas Myc silencing promotes rapid and complete tumor regression [7, 8]. In humans, Myc controls the expression of many genes including those, which play important roles in proliferation, survival, differentiation, ribosomal biogenesis, and cell metabolism [9]. This suggests that Myc levels must be maintained under tight control so as to minimize any potentially deleterious impact.

F-box and WD repeat domain containing 7 (FBXW7) is an E3 ubiquitin ligase that targets Myc for proteasomal degradation, when Myc is phosphorylated at the Thr58 (T58) residue and its phosphorylation at Ser62 (S62) must be removed by PP2A prior to binding by FBXW7 [10-12]. Human FBXW7 gene encodes three transcripts (FBXW7 $\alpha$, $\beta$, and $\gamma$ ) that only vary at the $\mathrm{N}$-terminus [13]. These isoforms have different subcellular location restricting interactions with specific partners and their functions. FBXW7 $\alpha$, 
$\beta$, and $\gamma$ locate in nucleoplasm, cytoplasm, and nucleolus, respectively [14], and FBXW7 $\alpha$ mRNA is expressed at much higher levels than FBXW7 $\beta$ and FBXW7 $\gamma$ in most human tissues, which suggests that FBXW7 $\alpha$ may play more important roles in physiological process than other isoforms [13]. In this study, we demonstrate a mechanistic link between the mitogen-activated kinase kinase kinase 13 (MAP3K13), tripartite motif containing 25 (TRIM25), FBXW7 $\alpha$, Myc and HCC development.

MAP3K13 is a member of the serine/threonine protein kinase family that regulates NF-KB [15], JNK [16, 17], and mutant p53 [18] pathways, all of which play vital roles in oncogenesis. TRIM25 is a member of the tripartite motif (TRIM) family and is aberrently expressed in many cancers such as lung cancer [19, 20], gastric cancer [21], ovarian cancer [22], colorectal cancer [23], and breast cancer [24]. It has been shown to interact with and degrade MTA-1 [25], which promotes proliferation in HCC.

Our previous work discovered the link between expression of MAP3K13 and Myc [26] and our laboratory has been focused on the pathogenesis of HCC. Therefore, we further strengthen the link between MAP3K13 expression and Myc stabilization in HCC. Here, we show that MAP3K13 upregulation correlates with HCC patient survival. Mechanistically, MAP3K13 phosphorylates and stabilizes TRIM25 by decreasing its polyubiquitination and proteasomal degradation, while TRIM25 ubiquitinates and degrades FBXW7 $\alpha$, a major E3 ubiqutin ligase of Myc, thereby stabilizing Myc so as to promote HCC development. These results indicate that MAP3K13 is a positive albeit indirect regulator of Myc. Our study identifies MAP3K13-TRIM25-FBXW7 $\alpha$ signaling axis as a novel potential target of treatment in Myc overexpressing human cancers.

\section{Results}

\section{MAP3K13 enhances Myc protein stability}

To assess whether MAP3K13 affects Myc expression, MAP3K13 depletion was performed in HCC HepG2 and FHCC98 cells using specific short hairpin RNAs (shRNAs) (Fig. 1a). MAP3K13 depletion did not affect Myc mRNA levels but decreased its protein abundance (Fig. 1a, b), suggesting that MAP3K13 may positively regulate Myc protein levels post-transcriptionally. Similarly, a pulsechase analysis performed following cycloheximide block of de novo protein synthesis revealed that MAP3K13 depletion significantly shortened the endogenous Myc protein half-life in the above cells (Fig. 1c, d), while ectopic MAP3K13 expression had the opposite effect (Fig. S1a, b), further supporting that MAP3K13 could enhance Myc protein stability.
Next, we sought to explore whether MAP3K13 could regulate the expression of Myc target genes. Our previous work indicated that ectopic MAP3K13 expression markedly increased Myc target transcript levels [26]. Consistent with this, gene-set enrichment analysis (GSEA) clearly demonstrated that Myc target genes were upregulated in samples expressing high levels of MAP3K13 in HCC databases from GSE102083 and GSE25097 (Fig. 1e, Table S1). Taken together, our results show that MAP3K13 play vita roles in regulating Myc stability and transcription activities.

\section{MAP3K13 stabilizes TRIM25}

Since MAP3K13 functions as a protein kinase, we next asked whether MAP3K13 could directly phosphorylate Myc. Because a direct interaction between MAP3K13 and Myc in vitro was not detected by GST pull-down assay (data not shown), we speculated that MAP3K13's regulation of Myc turnover was indirect and likely involved one or more intermediates. To this end, we expressed FLAGtagged MAP3K13 in HEK393T cells, immunoprecipitated the epitope-tagged protein and analyzed the precipitate by Mass Spectrometry (MS) (Fig. 2a). Although previous studies had shown that several ubiquitin E3 ligases, including FBXW7 [10-12], SKP2 [27, 28], and FBXL14 [29] regulated proteasome-mediated Myc degradation, none of these was identified by our MS analysis. However, the analysis did identify the E3 ubiquitin ligase TRIM25 as a candidate MAP3K13 interactor (Fig. 2a). An interaction between endogenous MAP3K13 and TRIM25 was further validated in HEK293T cells (Fig. 2b).

To map the region(s) of MAP3K13 responsible for its interaction with TRIM25, full-length TRIM25 and two deletion mutants of MAP3K13 were co-expressed (Fig. 2c). Subsequent co-immunoprecipitation assays revealed that the N-terminal kinase domain of MAP3K13 was solely responsible for the interaction with TRIM25 (Fig. 2c). Similar experiments performed with TRIM25 mutants showed that the RING domain was entirely responsible for the interaction with MAP3K13 (Fig. 2d).

We then asked whether MAP3K13 affects the stability of TRIM25. A time-course analysis following cycloheximide block indicated that MAP3K13 depletion significantly shortened the half-life of endogenous TRIM25 in HepG2 and FHCC98 cells (Fig. 2e, f). Together, these results indicate that MAP3K13 interacts with and stabilizes TRIM25.

\section{MAP3K13 phosphorylates TRIM25 at S12 and decreases its polyubiquitination to promote HCC growth}

Based on the fact that MAP3K13 is a serine/threonine protein kinase that interacts with and stabilizes TRIM25, we 


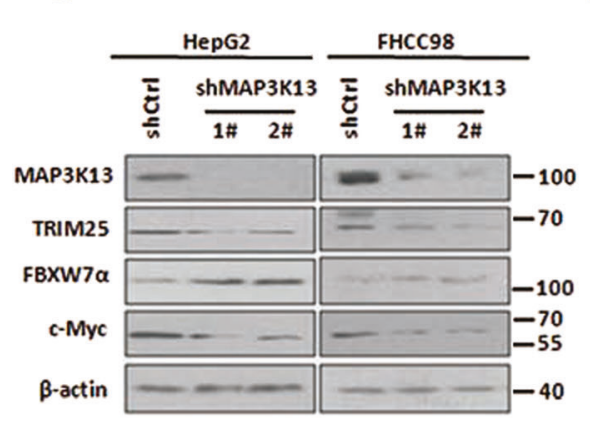

b

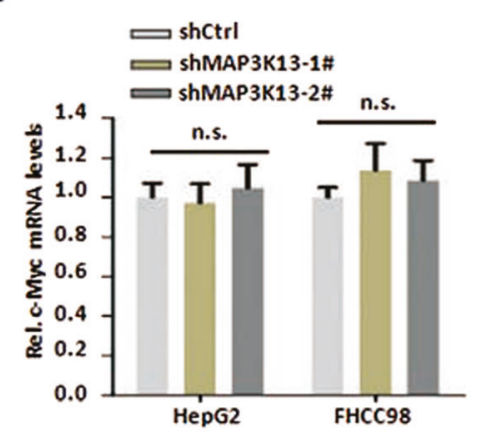

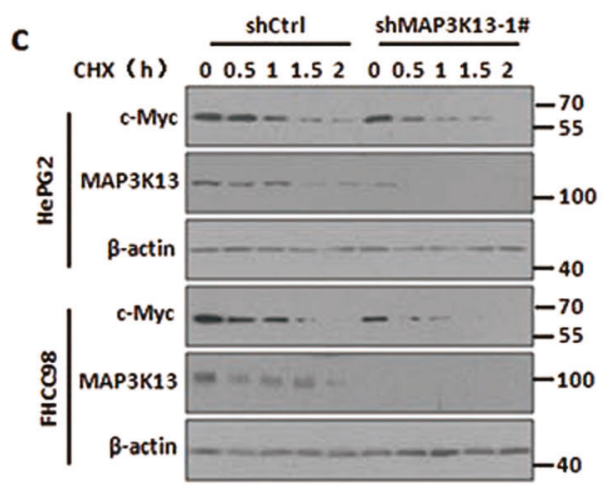

d

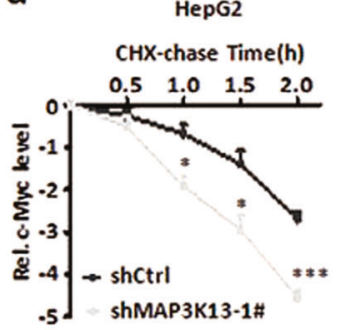

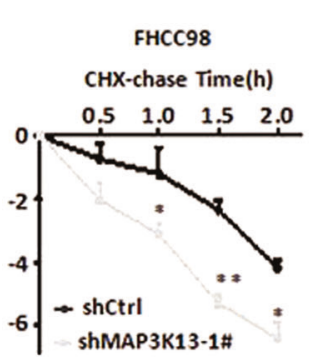

Fig. 1 MAP3K13 increases Myc stability and transcriptional activity in HCC. a Protein levels of MAP3K13, TRIM25, FBXW7 $\alpha$ and Myc in MAP3K13 shRNA-transfected HepG2 and FHCC98 cells were detected by immuno-blot. $\beta$-actin levels were used as a loading control. b Real-time qRT-PCR analysis of Myc expression in the above cells. Data were presented as the mean $\pm \mathrm{SD}, * p<0.05$. c MAP3K13 regulates Myc turnover. HepG2 and FHCC98 cells were transfected with MAP3K13 shRNA or control vectors followed by cycloheximide (CHX) block and collected at the indicated times for immuno-blot. e
c-Myc signature
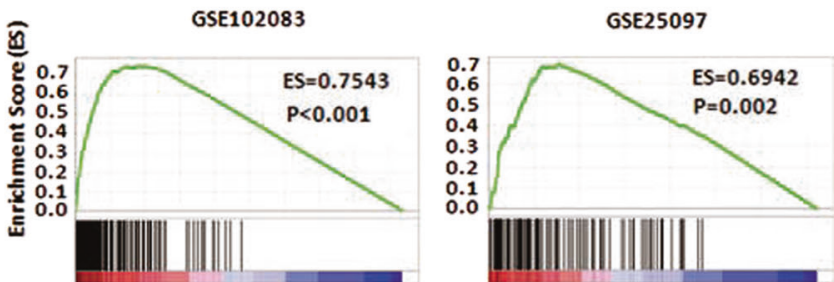

d The intensity of Myc expression for each time point in $\mathbf{c}$ was quantified by densitometry, with $\beta$-actin as a normalizer. Data were presented as the mean $\pm \mathrm{SD}, * p<0.05, * * p<0.01, * * * p<0.001$. e Gene-set enrichment analysis (GSEA) of MAP3K13 in HCC patients from GSE102083 and GSE25097. Representative GSEA plots indicated that predefined gene sets involved in the Myc pathway were positively associated with high MAP3K13 expression. ES enrichment score

the wild-type (WT) TRIM25 (Fig. S2b, c). Therefore, functionally relevant phosphorylation by MAP3K13 could be occurring at S12, S14, or both sites of TRIM25, which was consistent with the previous result that MAP3K13 phosphorylated TRIM25 only at serine residues (Fig. 3a). To identify the precise phosphorylation site(s), the nonphosphorylatable individual S12A and S14A mutants were constructed and again co-expressed with MAP3K13. Our results showed that MAP3K13 was unable to stabilize only the TRIM25 S12A mutant protein (Fig. 3b), thus implicating this as the functionally relevant site. To further validate this, a pan phospho-serine antibody was used to evaluate serine phosphorylation of TRIM25 and the S12A mutant. As expected, MAP3K13 failed to increase the phosphorylation level of the TRIM25 S12A mutant (Fig. 3c) and this was confirmed by kinase assays in vitro (Fig. 3d). Overall, TRIM25 S12A was expressed at a lower level than WT and other mutant TRIM25 proteins (Fig. S2d), thus supporting the notion that this residue plays a critical role in maintaining protein stability as did the finding that the TRIM25 S12 residue and its neighboring amino acids are evolutionarily conserved among multiple species (Fig. 3e). 
a

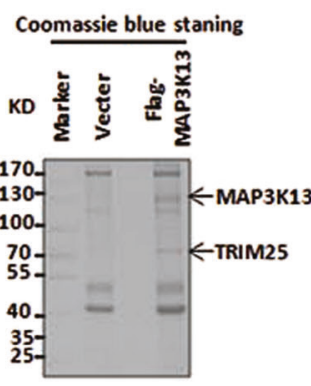

\begin{tabular}{cc}
\hline Protein & Number of peptides \\
\hline TRIM25 & 22 \\
TKT & 20 \\
RANGAP1 & 19 \\
LRRC47 & 18 \\
RPN1 & 18 \\
GNL3 & 17 \\
SAMHD & 15 \\
\hline
\end{tabular}

b

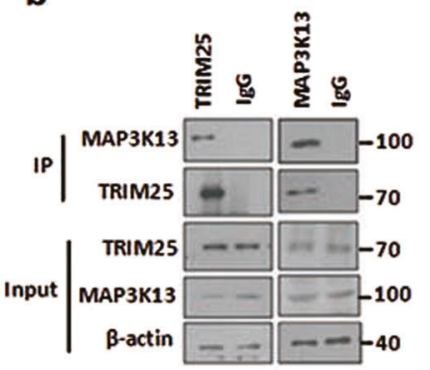

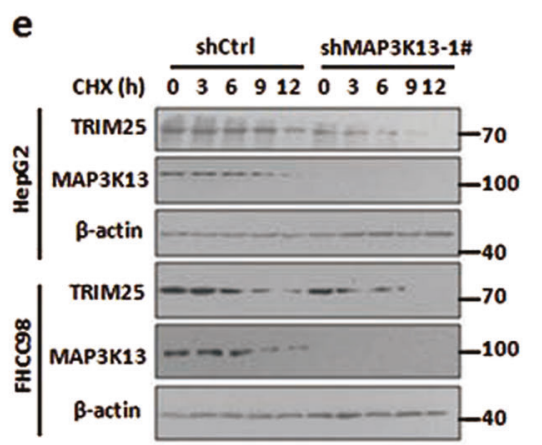

f
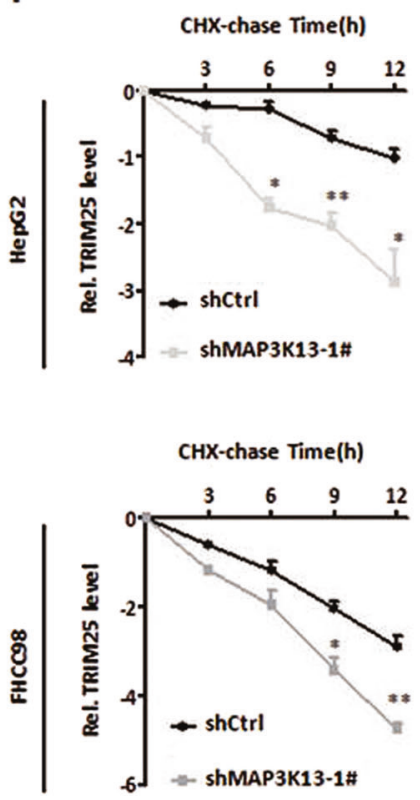

Fig. 2 MAP3K13 stabilizes TRIM25. a TRIM25 was identified as MAP3K13-interacting protein. Cellular extracts from HEK293T cells expressing Flag-MAP3K13 and control vector were immunopurified with anti-FLAG affinity columns. The eluates were resolved by SDSPAGE and Coomassie blue staining. The protein bands were retrieved and analyzed by mass spectrometry. b Endogenous interaction between MAP3K13 and TRIM25. c, d Schematic diagram showed the structure of MAP3K13 (left) and TRIM25 (right) and truncation mutants used. Flag-tagged MAP3K13 WT or truncation mutants were co-expressed with HA-TRIM25 in HEK293T. Extracts were immunoprecipitated with Flag antibody and examined by Western blotting. HA-tagged TRIM25 WT or truncation mutants were coexpressed with Flag-tagged MAP3K13 in HEK293T (c). Co-IP with HA antibody and examined by indicated antibodies (d). e MAP3K13 regulates TRIM25 protein turnover. HCC HepG2 and FHCC98 cells transfected with the indicated plasmids were treated with $\mathrm{CHX}$ and collected at the indicated times for immuno-blot. $\beta$-actin was used as a loading control. f The intensity of TRIM25 expression for each time point in e was quantified by densitometry, with $\beta$-actin as a normalizer. Data were presented as the mean $\pm \mathrm{SD},{ }^{*} p<0.05 ; * *<0.01$
To further test the role of the TRIM25 S12 residue, the phosphomimetic aspartic acid mutant S12D was constructed. Cycloheximide chase experiments were then performed to estimate the half-lives of WT TRIM25 and its mutants (S12A and S12D). As expected the TRIM25 S12A mutant had a shortened half-life, whereas the S12D mutant had a significantly extended half-life (Fig. 3f, g). Collectively, these studies showed that MAP3K13-mediated phosphorylation of the TRIM25 S12 site is a critical determinant of the latter protein's stability.

Because of the location of the critical S12 residue within the TRIM25 RING finger domain, we hypothesized that phosphorylation of this amino acid might alter ubiqutination and proteasomal degradation. Indeed, an in vivo ubiquitination assay suggested that the TRIM25 S12A mutant was more highly polyubiqutinated compared to WT TRIM25 and its mutant S12D (Fig. S2e). TRIM25 has been reported to auto-ubiquitylate itself [30]. We reasoned that phosphorylation by MAP3K13 might change the E3 ubiquitin ligase activity of TRIM25 toward itself, leading to decreased self-polyubiquitination. To elucidate our notion, we constructed an E3 ligase-defective TRIM 25 mutant by mutating cysteines 50 and 53 to serine (TRIM25 dead), which loses E3 ubiquitin ligase activity [31]. In vivo ubiquitination assays showed that overexpression of MAP3K13 could markedly decrease WT TRIM25 

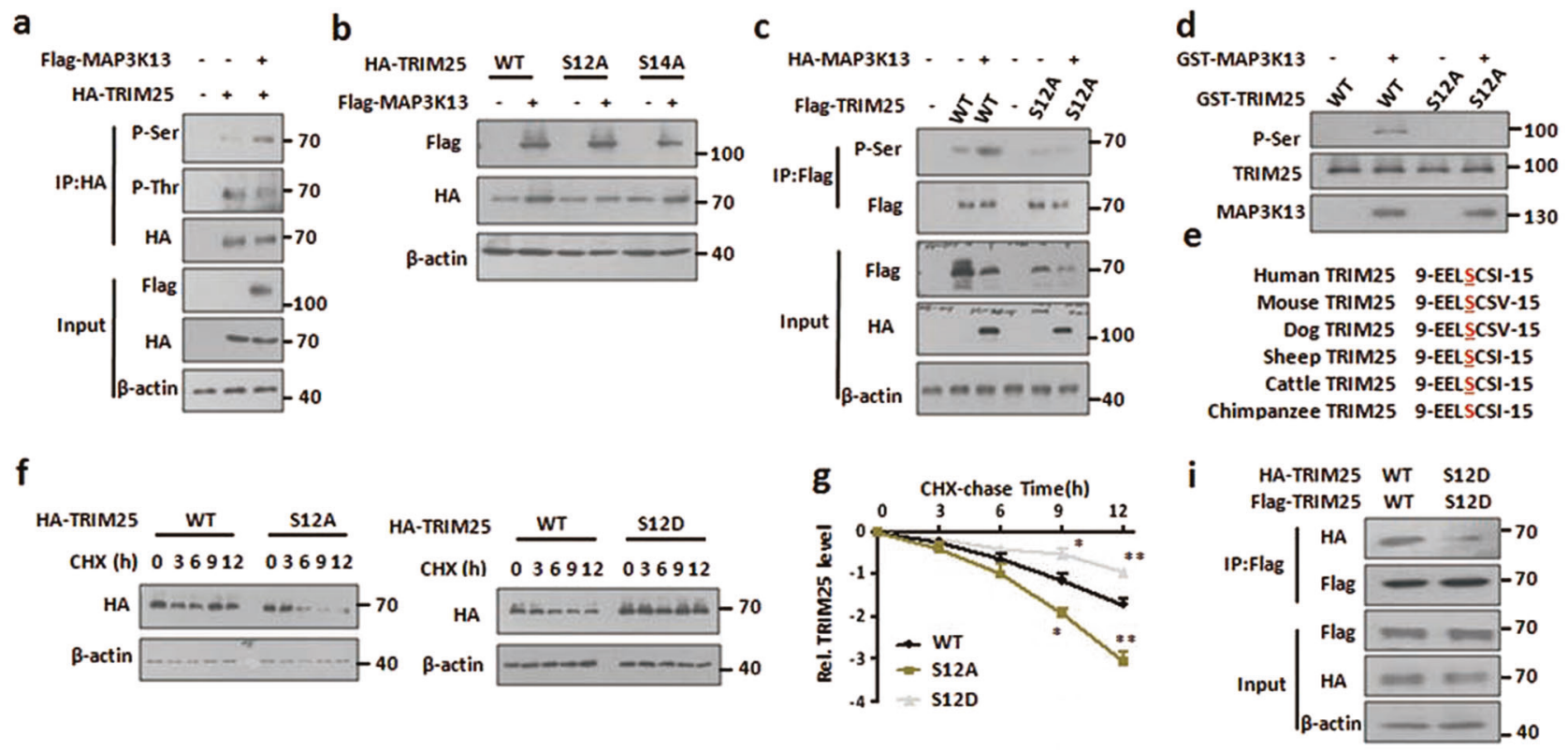
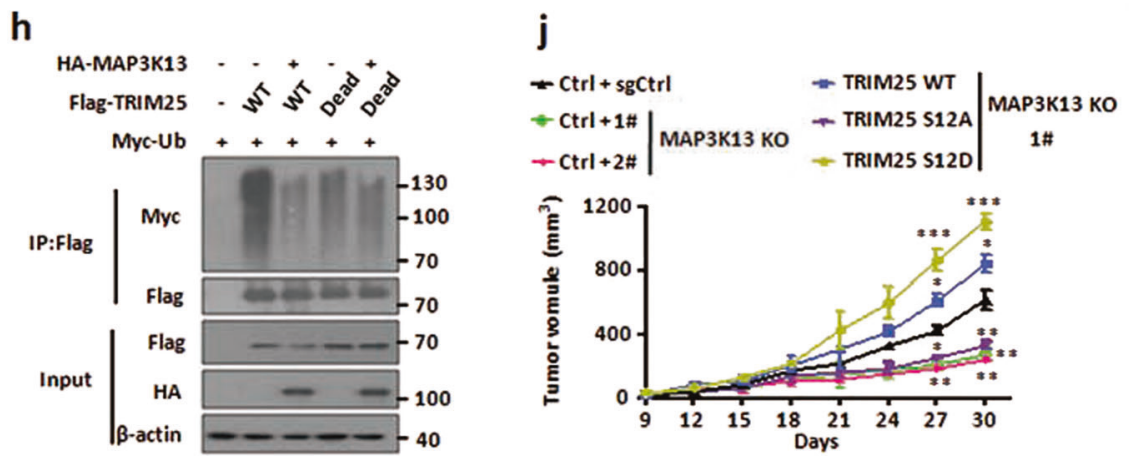

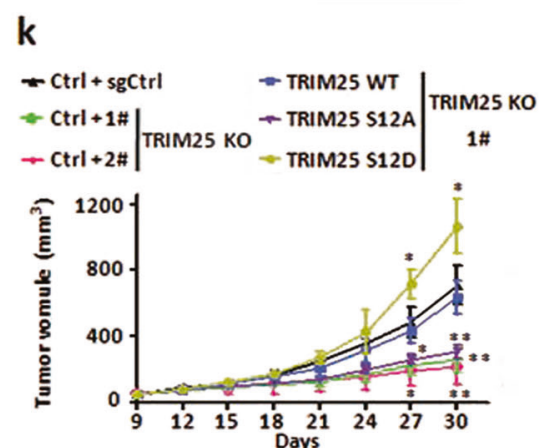

Fig. 3 MAP3K13 phosphorylates TRIM25 at Ser $_{12}$. a MAP3K13 phosphorylates TRIM25 at serine residue(s). Immunoprecipitation of HA-TRIM25 with anti-HA-agarose beads was following immuno-blot with the indicated antibodies. The p-Ser and p-Thr denote pan phospho-serine and pan phospho-threonine antibodies, respectively. b MAP3K13 doesn't stabilize TRIM25 S12A mutant protein. HEK293T cells were transfected with the indicated plasmids and $48 \mathrm{~h}$ later, cells were harvested for immuno-blots. c, d MAP3K13 phosphorylates TRIM25 at $\mathrm{Ser}_{12}$ in vivo and in vitro. Immuno-precipitation of Flag-TRIM25 with anti-FLAG-agarose from extracts of HEK293T cells transfected with the indicated plasmids and subsequent immuno-blots with the indicated antibodies (c). In vitro kinase reaction was performed by incubating recombinant GST-MAP3K13, and GSTTRIM25 WT or GST-TRIM25 S12A protein and subsequent immunoblots with indicated antibodies (d). e Amino acid sequences of TRIM25 from the indicated species are highly conserved around the
$\mathrm{Ser}_{12}$ phosphorylation site. f TRIM25 S12D mutant had a significantly extended half-life. HEK293T cells were transfected with the indicated plasmids, treated with $\mathrm{CHX}$ and harvested at the indicated time points for immuno-blot. $g$ The intensity of TRIM25 expression for each time point in $\mathbf{f}$ was quantified by densitometry, with $\beta$-actin as a normalizer. Data were presented as the mean $\pm \mathrm{SD},{ }^{*} p<0.05, * * p<0.01$. h MAP3K13 decreases polyubiquitination of WT TRIM25, but not that of TRIM25 dead mutant. HEK293T cells were transfected with the indicated plasmids, treated with MG132 for $6 \mathrm{~h}$ and then lysed in RIPA buffer. Immuno-precipitation of ubiquitin-conjugated TRIM25 proteins with anti-FLAG affinity agarose and subjected to immunoblot assay with Myc tag antibody. j, k Subcutaneous xenograft experiments were performed in FHCC98 MAP3K13 KO cells (j) or FHCC98 TRIM25 KO cells (k) stably expressing the indicated plasmids $(n=3)$. Data were presented as the mean $\pm \mathrm{SD}, * p<0.05$, $* * p<0.01, * * * p<0.001$ polyubiquitination but not TRIM25 dead mutant (Fig. 3h). Also the TRIM25 S12D mutant exhibited an attenuated ability to form dimerization (Fig. 3i). MAP3K13 depletion markedly restrained the proliferation of HCC HepG2 and FHCC98 cells and this could be rescued by ectopic expression of WT TRIM25 and its S12D mutant but not by the S12A mutant (Fig. S2f, g). To investigate the effect of S12 phosphorylation of TRIM25 in vivo, MAP3K13, and TRIM25 knockout (KO) FHCC98 cells and a series of stable cell lines were constructed and subcutaneously injected into the flanks of nude mice. MAP3K13 and TRIM25 KO decreased growth of xenografts derived from FHCC98 cells, which were substantially rescued by the expression of TRIM25 WT and its S12D mutant, but not its S12A mutant (Fig. 3j, k, Fig. S2h-k). Collectively, these results reveal that MAP3K13 phosphorylates and stabilizes TRIM25 through decreasing its polyubiquitination to promote tumor grown in vivo. 


\section{TRIM25 increases Myc protein stability}

Since TRIM25 is one of MAP3K13's downstream target proteins, we investigated whether TRIM25 could also regulate Myc stability. TRIM25 depletion by shRNAs in HepG2 and FHCC98 cells caused a significant decline in Myc protein abundance (Fig. 4a), without affecting mRNA levels (Fig. 4b). These results suggested that TRIM25 regulates Myc expression by a post-translational mechanism.

TRIM25 enhanced Myc protein stability by decreasing its polyubiquitination (Fig. 4c). Time-course analysis also showed that TRIM25 overexpression extended the halflife of endogenous Myc in HEK293T cells (Fig. 4d, e). To better test Myc protein stability, the Myc open reading frame (ORF) was fused to the C-terminus of firefly luciferase to produce the fusion protein (Luc-Myc), which was used as a reporter of protein stability in the presence of WT TRIM25 or its mutants. WT TRIM25 and its partial mutants (T25/26 A, S35/T40/46 A, S14A) also significantly increased Luc-Myc activity (Fig. 4f). Of note, TRIM25 S12D was associated with the highest Luc-Myc activity, whereas TRIM25 S12A was associated with the lowest activity (Fig. 4f). An in vivo ubiquitination assay indicated that TRIM25 S12D and S12A, respectively, decreased and increased endogenous Myc ubiquitylation levels relative to the WT TRIM25 protein (Fig. 4g). GSEA clearly showed that TRIM25 also reglulated Myc targets in HCC databases from GSE102083 and GSE25097 (Fig. 4h, Table S2). These results indicate that MAP3K13-dependent TRIM25 S12 phosphorylation enhances Myc protein stabilization.

\section{TRIM25 abrogates FBXW7a-mediated Myc degradation to promote HCC cell proliferation}

E3 ubiquitin ligases target numerous proteins for ubiquitinmediated degradation [32, 33]. Therefore, we speculated that the E3 ubiquitin ligase TRIM25 might degrade a negative regulator of Myc. Several expression vectors for Myc E3 ubiquitin ligases (FBXW7 $\alpha$, SKP2, and FBXL14) and its negative regulator GSK3 $\beta$ were thus tested in a series of dual-luciferase report system assays in HEK293T cells. Consistent with previous studies, all three E3 ubiquitin ligases and GSK3 $\beta$ significantly decreased Luc-Myc activity (Fig. 5a). Of note was that, compared with other combinations, only that of TRIM25 and FBXW7 $\alpha$ restored the original levels of Luc-Myc activity (Fig. 5a), thus supporting the idea that TRIM25 acts specifically on FBXW7 $\alpha$. In line with this, FBXW7 $\alpha$ caused a dramatic decline in Myc protein levels, which was rescued by the co-expression of TRIM25 and FBXW7 $\alpha$ (Fig. 5b). Moreover FBXW7 $\alpha$ markedly enhanced endogenous Myc ubiquitination, whereas the co-expression of TRIM25 with FBXW7 $\alpha$ significantly suppressed this (Fig. 5c).

TRIM25 depletion also markedly inhibited the proliferation of both HepG2 and FHCC98 cells whereas FBXW7 $\alpha$ depletion had the opposite effect (Fig. 5d, e). Most notably, compared with control counterpart, codepletion of TRIM25 and FBXW7 $\alpha$ restored a nearly normal growth rate (Fig. 5d, e), thus further validating the idea that TRIM25 promotes Myc stabilization via antagonizing FBXW7 $\alpha$-mediated degradation to promote HCC cell proliferation.

\section{TRM25 interacts with and ubiquitinates FBXW7 $a$ at K412}

TRIM25 abrogated FBXW7 $\alpha$ 's de-stabilization of Myc, urging us to speculate that TRIM25 might degrade FBXW7 $\alpha$, but not other Myc E3 ligases (FBXL14, SKP2) or GSK3 $\beta$. To test this, FBXW7 $\alpha$, FBXL14, SKP2, and GSK3 $\beta$ were each fused to the C-terminus of firefly luciferase and the resultant fusion proteins were used as reporters of the indicated protein's stability in the presence of TRIM25. The data showed that ectopic TRIM25 expression decreased the luciferase activity only of LucFBXW7 $\alpha$ (Fig. S3a). TRIM25 expression also significantly reduced the protein levels of FLAG-tagged FBXW7 $\alpha$ in a dose-independent manner and TRIM25-mediated FBXW7 $\alpha$ downregulation was rescued by MG132, a $26 \mathrm{~S}$ proteasome inhibitor (Fig. S3b). TRIM25 depletion by shRNAs in HepG2 and FHCC98 cells significantly increased the protein levels of FBXW7 $\alpha$, but not that of FBXW7 $\beta$ or FBXW7 $\gamma$ (Fig. S3c). Because TRIM25 could modulate FBXW7 $\alpha$ stability, we examined their direct interaction in HEK293T cells and demonstrated this by coimmunoprecipitation (Co-IP) (Fig. 6a). We then mapped the region of FBXW7 $\alpha$ responsible for the TRIM25 using three deletion mutants (Fig. 6b). Co-IP assays revealed that the C-terminal WD40 domain of FBXW7 $\alpha$ was responsible for the interaction with TRIM25 (Fig. 6b). Conversely, the C-terminal SPRY domain of TRIM25 was necessary for the FBXW7 $\alpha$ interaction (Fig. 6c). As expected, TRIM25 depletion significantly extended the half-life of FBXW7 $\alpha$ in HepG2 and FHCC98 cells in response to a cycloheximide block (Fig. 6d, e) and ectopic TRIM25 expression markedly reduced the half-life of FBXW7 $\alpha$ in HEK293T cells (Fig. S3d-g).

Next, we asked whether TRIM25 could ubiquitinate and affect the stability of FBXW7 $\alpha$. The ectopic expression of TRIM25 significantly increased FBXW7 $\alpha$ ubiquitination levels in a dose-independent manner (Fig. S3h). As FBXW7 is also known to auto-ubiquitylate [34, 35], it could be possible that interaction with TRIM25 promotes the autoubiquitylation of FBXW7 $\alpha$, not ubiquitylating it directly. 


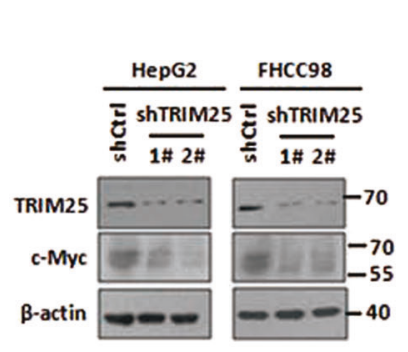

f

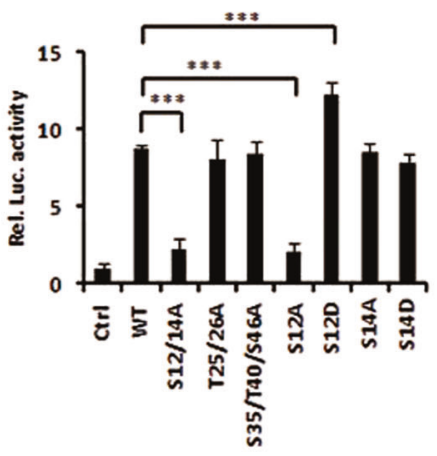

b

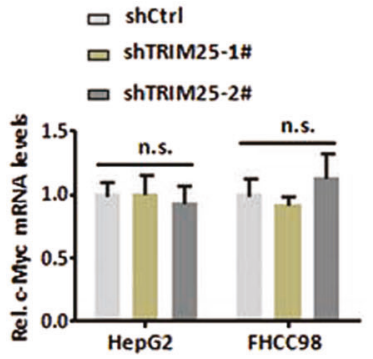

g

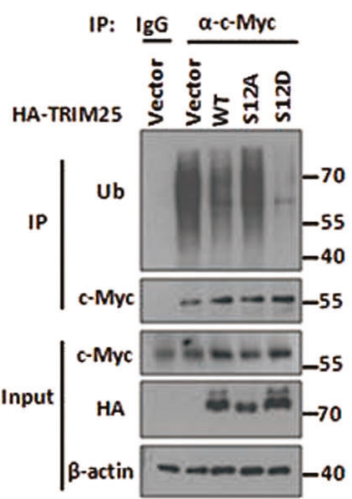

\section{C}

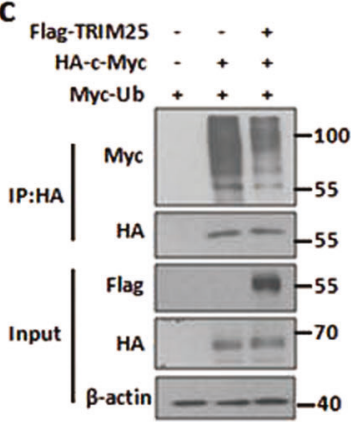

d

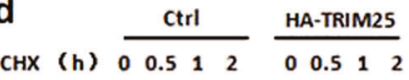

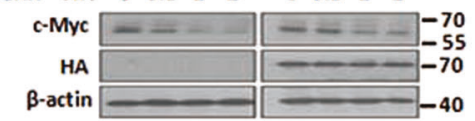

e

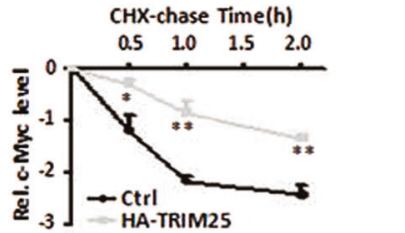

h

\section{c-Myc signature}
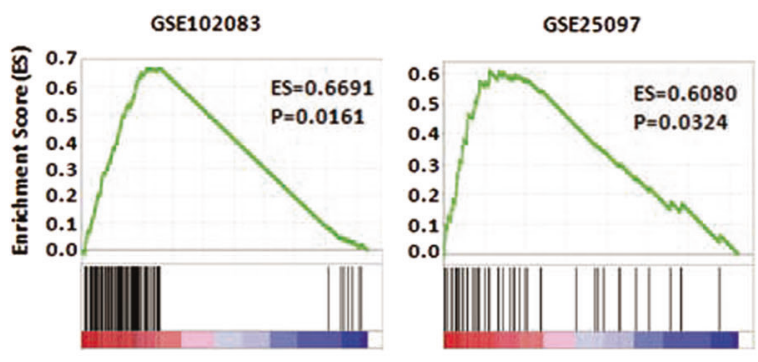

Fig. 4 TRIM25 stabilizes Myc. a Relative expression of TRIM25 and Myc in TRIM25 shRNA-transfected HCC cells HepG2 and FHCC98. b Real-time qRT-PCR analysis of Myc transcript expression in the above cells. Data were presented as the mean $\pm \mathrm{SD}, * p<0.05$. c TRIM25 decreases Myc ubiquitination. HEK293T cells were transfected with the indicated plasmids and then treated with MG132 for $6 \mathrm{~h}$ and then lysed in RIPA buffer followed by immunoprecipitation and immuno-blotting as described in Fig. 3h. d Ectopic expression of TRIM25 increases Myc protein levels. HEK293T cells were transfected with HA-TRIM25 or control vectors, treated with CHX and collected at the indicated times for immuno-blot. e The intensity of Myc expression for each time point in d was quantified by

To eliminate the possiblity, we constructed an E3 ligasedefective FBXW7 $\alpha$ mutant by mutating arginine 465 to histidine (FBXW7 $\alpha$ dead), based on its high frequency in human cancers as well as its loss-of-function in substrate binding activity [36]. An in vivo ubiquitination assay suggested that TRIM 25 could markedly increase WT FBXW7 $\alpha$ and FBXW7 $\alpha$ dead mutant polyubiquitination but TRIM25 dead mutant failed to do that (Fig. 6f). Next, to determine the type of TRIM25-mediated ubiquitination of FBXW7 $\alpha$, vectors expressing several Myc-tagged ubiquitin mutants (K6O, K11O, K27O, K29O, K33O, K48O, and K63O), which contained substitutions of arginine for all lysine resides except the lysine at this position, respectively, were used. The data showed that ubiquitination of FBXW7 $\alpha$ was found only in the presence of WT and K48O Ubiquitin, suggesting that TRIM25 mediated K48-linked ubiquitination of FBXW7 $\alpha$, but not other position-linked ubiquitination of FBXW7 $\alpha$ (Fig. $6 \mathrm{~g}$ ). densitometry, with $\beta$-actin as a normalizer. Data were presented as the mean $\pm \mathrm{SD}, * p<0.05, * * p<0.01$. f TRIM25 S12D mutant significantly increases Luc-Myc activity. The Luc-Myc reporter plasmid was transfected with WT TRIM25 or its mutants expression vectors into HEK293T cells. $24 \mathrm{~h}$ later, dual-luciferase assays were performed. Data were presented as the mean $\pm \mathrm{SD}, * * p<0.01$, $* * * p<0.001$. g TRIM25 S12D mutant significantly decreases polyubiquitination of endogeous Myc. h GSEA of TRIM25 in HCC patients from GSE102083 and GSE25097. Representative GSEA plots indicated that predefined gene sets involved in the Myc pathway were positively associated with high TRIM25 expression. ES enrichment score

Further, we sought to explore the FBXW7 $\alpha$ sites ubiquitinated by TRIM25. Interestingly, ectopic TRIM25 expression significantly decreased the protein levels of two FBXW7 $\alpha$ 's depletion mutants $(\Delta \mathrm{F}-$ box and WD40) in a dose-independent manner, whereas it failed to decrease the protein levels of $\Delta$ WD40 mutant (Fig. S4), implying that TRIM25 ubiquitinated FBXW7 $\alpha$ within its WD40 domain. As expected, TRIM25 dead mutant could not decrease the protein levels of all mutants, suggesting that TRIM25's E3 ligase activity was needed to degrade FBXW7 $\alpha$ (Fig. S4). One candidate site, the evolutionarily conserved K412 residue, was found in the Phospho-Site Plus database (https://www.phosphosite.org) (Fig. 6h). Ectopic TRIM25 expression markedly enhanced the levels of ubiquitinated WT FBXW7 $\alpha$, while failed to increase ubiquitination levels of mutant K412R (Fig. 6i). As expected, cycloheximide chase experiments show FBXW7 $\alpha$ K412R mutant had a much significantly extended half-life than WT FBXW7 $\alpha$ 

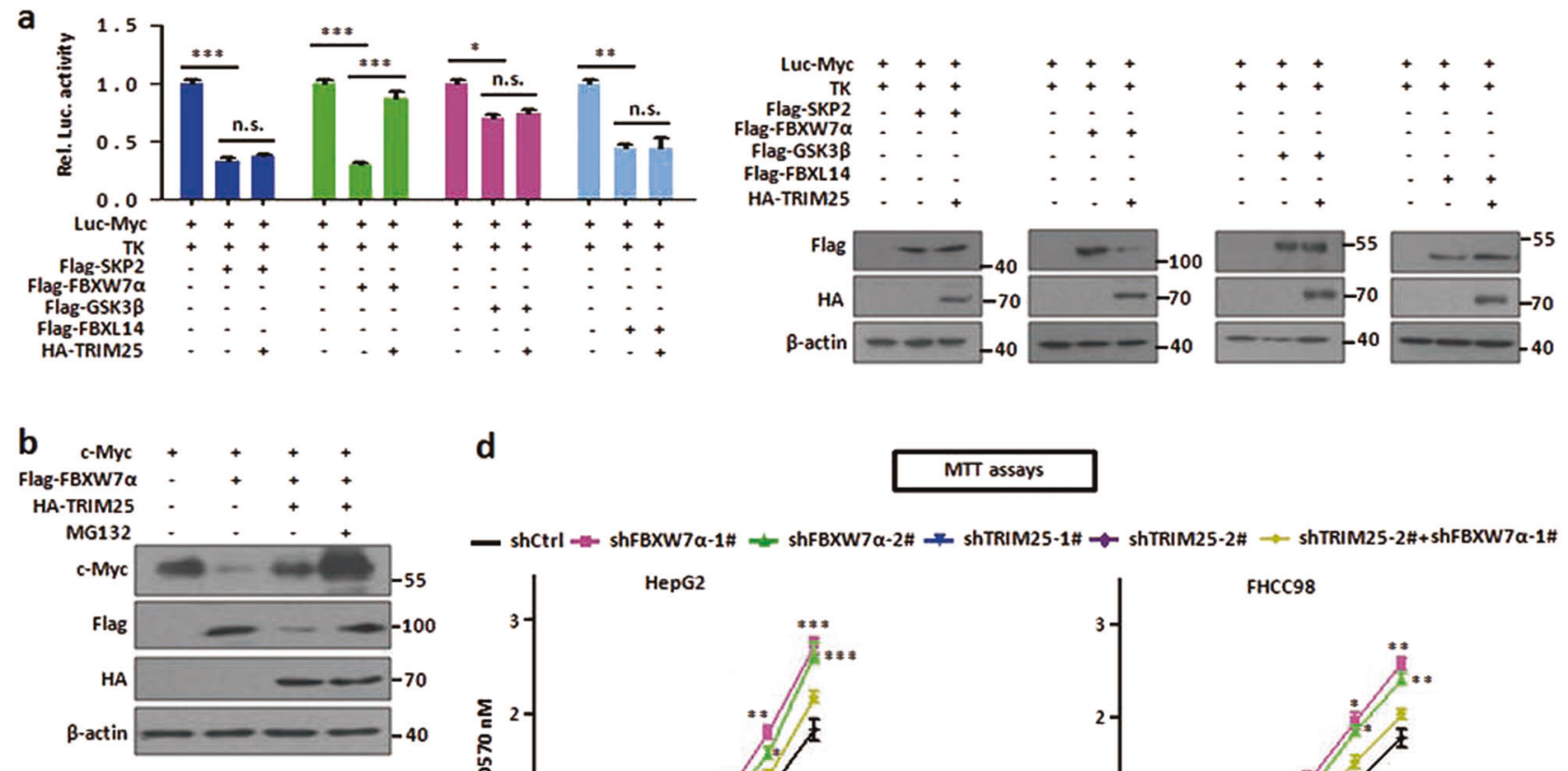

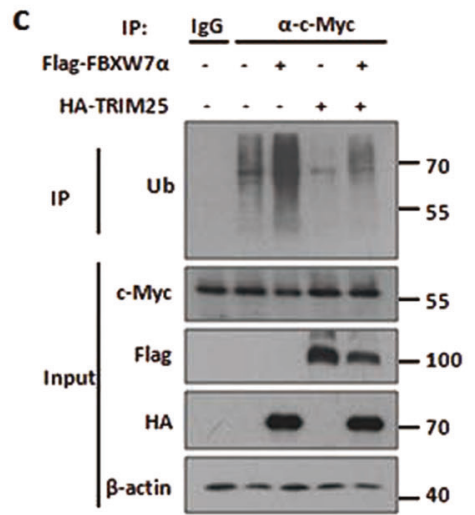

Fig. 5 TRIM25 increases Myc stability via FBXW7 $\alpha$. a TRIM25 blocks FBXW7 $\alpha$ 's ability to stablize Myc. HEK293T cells were transfected with the indicated plasmids followed by the performance of dual-luciferase assays (left). The immuno-blot showed indicated protein levels of above cells (right). Data were presented as the mean $\pm \mathrm{SD}, * p<0.05, * * p<0.01, * * * p<0.001$. b HEK293T cells were transfected with the indicated plasmids and treated with MG132

(Fig. S5). These results revealed that TRIM25 ubiquitinates $\mathrm{FBXW} 7 \alpha$ at K412.

\section{Dysregulation of the MAP3K13-TRIM25-FBXW7a- Myc axis in HCC}

MAP3K13, TRIM25 and Myc were significantly upregulated in a panel of human HCC specimens, whereas FBXW7 was downregulated, compared with adjacent nontumor tissue (Fig. 7a). Similar findings were obtained in unpaired and paired HCC samples from the GEO databases (GSE102083, GSE25097, and GSE45436) (Fig. 7b, c) and unpaired GBM and PRAD samples from the TCGA database (Fig. S6). Further, upregulation of MAP3K13 for $6 \mathrm{hr}$ before being collected for immuno-blot. c TRIM25 stablizes endogenous Myc by decreasing FBXW7 $\alpha$-mediated ubiquitination. d MTT assays were performed in HepG2 and FHCC98 cells stably expressing the indicated plasmids. Data were presented as the mean \pm $\mathrm{SD}, * p<0.05, * * p<0.01, * * * p<0.001$. e The immuno-blot showed protein levels of cells from $\mathbf{d}$

transcript was also validated in $\mathrm{HCC}$ samples from the TCGA (Fig. 7d). As shown in Fig. 7e, the MAP3K13 gene was found to be significantly amplified in HCCs. MAP3K13 mRNA levels in HCCs also generally correlated with the degree of its gene amplification and gain (Log2 SNP >0), which suggests that dysregulated MAP3K13 expression is the result of gene amplification and gain (Fig. 7f). Also MAP3K13 upregulation strongly correlated with poor HCC patient Overall Survival (OS) in TCGA (Fig. 7g). Similarly, TRIM25 was highly expression in HCCs and this upregulation strongly correlated with poor OS in TCGA (Fig. 7h, i). Taken together, these data suggest that inhibition of the MAP3K13-TRIM25 signaling could be of potential therapeutic benefit. 
a

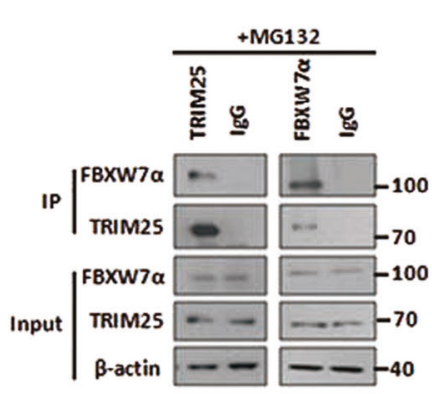

b

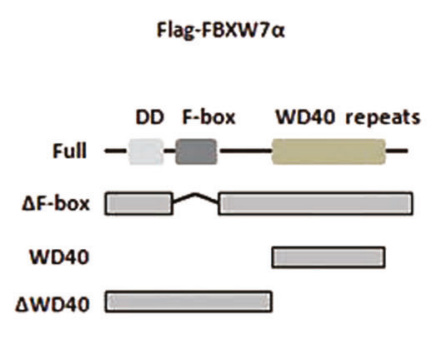

Flag-FBXW7a

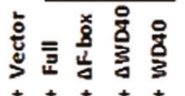

HA-TRIM2S +++++

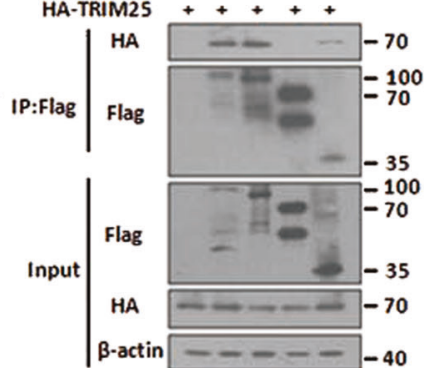

C

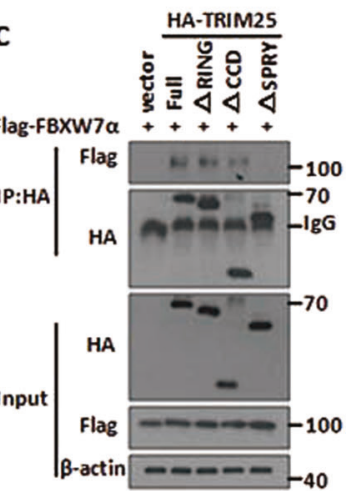

d

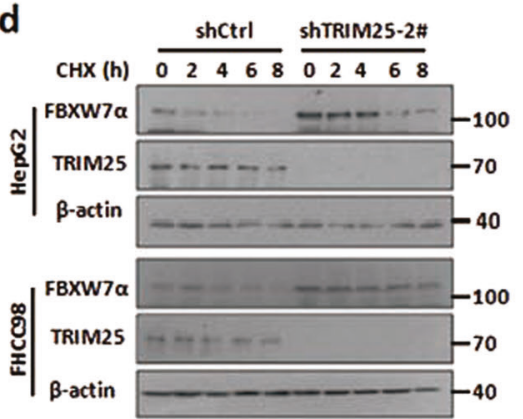

g e

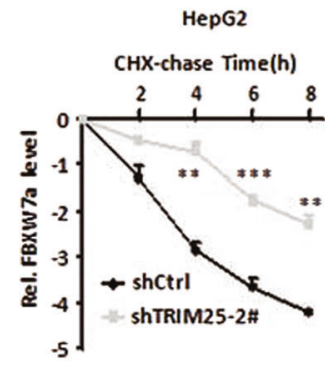

h

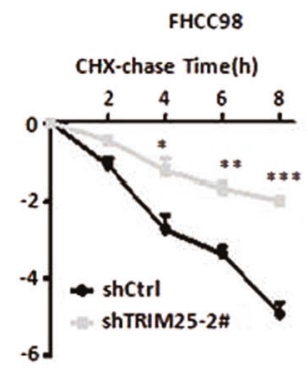

$f$ HA-TRIM25-WT $: \ldots+\cdots+\ldots$ Flag-FBXW7a-WT - + + . + Flag-FBXW7 $\alpha$-dead . . . + + +

Myc-Ub +++++++

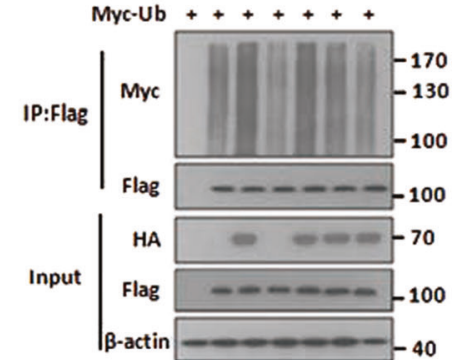

i

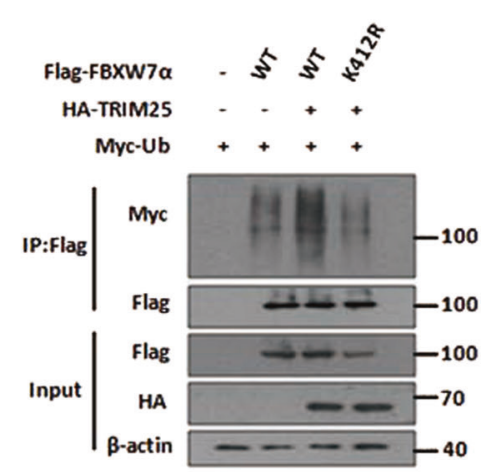

Fig. 6 TRIM25 ubiquitinates FBXW7 $\alpha$ at K412. a Endogenous interaction between TRIM 25 and FBXW7 $\alpha$. b, c TRIM25 binds to FBXW7 $\alpha$ via the WD40 and SPRY domains. Left: Schematic diagram of full-length FBXW7 $\alpha$ and its truncation mutants. Right: HEK293T cells co-transfected with HA-TRIM25 and FLAGFBXW $7 \alpha$ or its truncation mutants were immunoprecipitated with anti-FLAG-agarose and subsequently immuno-blotted with FLAG and HA antibodies (b). HEK293T cells co-transfected FLAG-FBXW7 $\alpha$ and HA-TRIM25 or its truncation mutants were immunoprecipitated with anti-HA agarose and subsequently immuno-blotted with FLAG and HA antibodies (c). d Inhibition of TRIM25 increases FBXW7 $\alpha$ stability. HepG2 and FHCC98 cells transfected with TRIM25 shRNA were treated with $\mathrm{CHX}$ and collected at the indicated times for

\section{Discussion}

HCC accounts for $\sim 70-90 \%$ of all primary liver cancers and is among the most common visceral neoplasms [37]. Recently the incidence of $\mathrm{HCC}$ has been increasing, whereas survival rates have remained very low [1]. Myc is often activated and immuno-blot. e The intensity of FBXW7 $\alpha$ expression for each time point in $\mathbf{d}$ was quantified by densitometry, with $\beta$-actin used as a normalizer. Data were presented as the mean $\pm \mathrm{SD},{ }^{*} p<0.05$, ${ }^{* *} p<$ $0.01, * * * p<0.001$. f TRIM25 increases polyubiquitination of WT FBXW7 $\alpha$ and FBXW7 $\alpha$ dead mutant. g TRIM25 promotes K48linked polyubiquitination of FBXW7 $\alpha$. HEK293T cells were cotransfected with FLAG-FBXW7 $\alpha$, HA-TRIM25 and Myc-Ub or its mutants as indicated and treated with MG132 followed by immunoprecipitation and immuno-blotting as described in Fig. $3 \mathrm{~h}$. h Conservation of amino acid sequences around ubiquitination sites of FBXW7 $\alpha$ are highly conserved among several species. i TRIM25 ubiquitinates FBXW7 $\alpha$ at K412

overexpression in HCCs thus making it an attractive and obvious therapeutic target. However, direct inhibition of Myc has been challenging [38]. An alternative approach is to inhibit Myc indirectly by focusing on its more tractable upstream regulators. These include the PLK1 and PIM1 kinases as well as the signalosome subunit CSN6, which are 


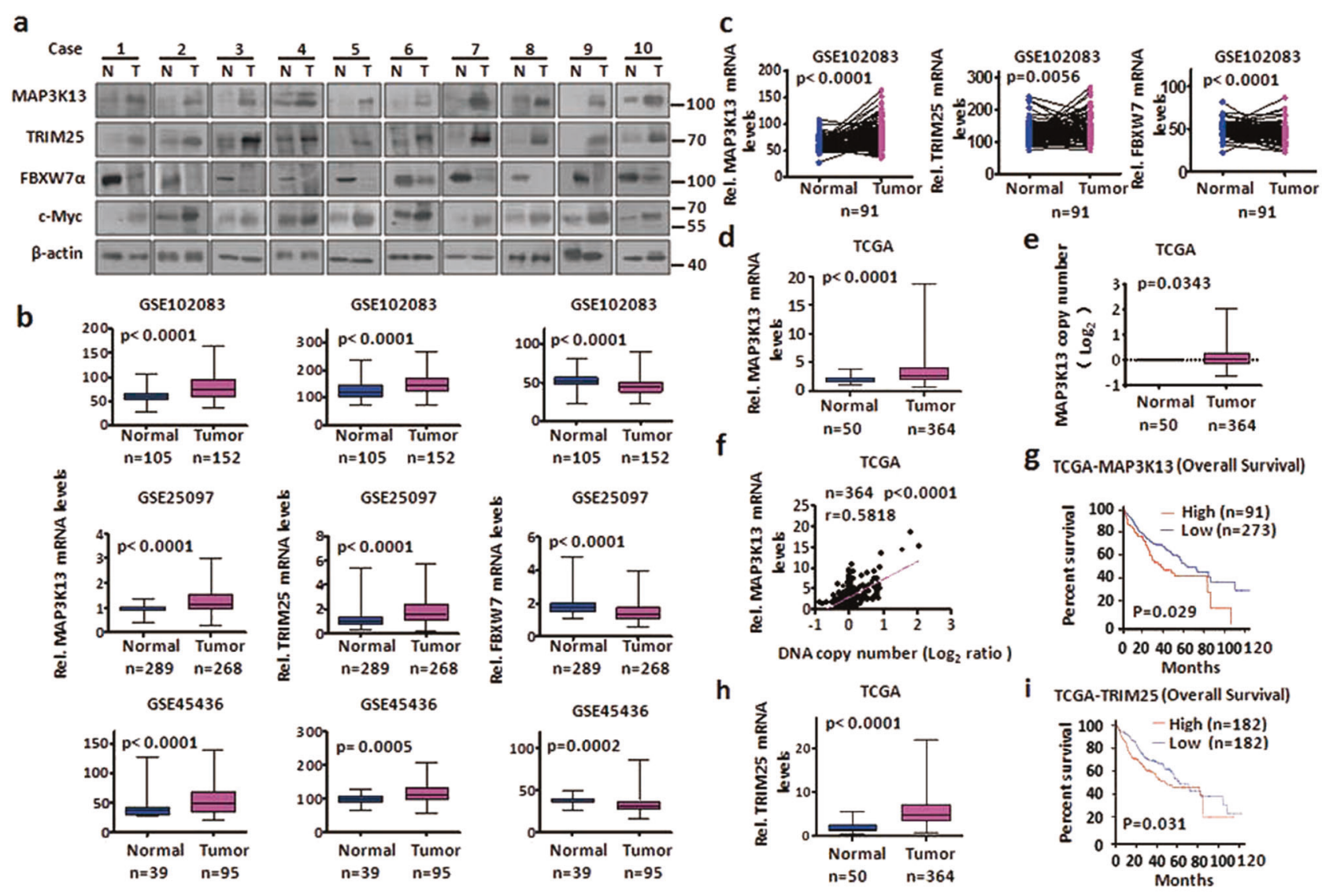

Fig. 7 Deregulation of the MAP3K13-TRIM25-FBXW7 $\alpha$ axis in human HCCs. a Protein levels of MAP3K13, TRIM25, FBXW7 $\alpha$ and Myc were detected in HCCs and their adjacent normal tissues by immuno-blot. b, c MAP3K13 and TRIM25 are upregulated, while FBXW7 $\alpha$ are downregulated in HCCs. Relative MAP3K13, TRIM25, and FBXW7 $\alpha$ mRNA levels in normal liver and HCCs from GSE102083, GSE25097, and GSE45436 (b). MAP3K13, TRIM25, and FBXW7 $\alpha$ mRNA levels were analyzed in paired HCC tissues from GSE102083 (c). d MAP3K13 mRNA levels in normal liver and

potentially druggable targets in several Myc-driven cancers [39-41]. In this study, we have shown the MAP3K13TRIM25 to be a particularly amenable therapeutic target in HCCs with Myc and perhaps even without Myc.

Although our previous study had determined that MAP3K13 increases the stability of Myc, the mechanism underlying this remained unclear [26]. Here, using a combination of Co-IP and mass spectrometry, we have found that MAP3K13 directly interacts with TRIM25. TRIM25 has been previously shown to play a crucial regulatory role by mediating K63-linked RIG-I polyubiquitination to induce antiviral responses [42]. c-Src phosphorylates TRIM25 at $\mathrm{Tyr}_{278}$, which is required for the complete activation of RIG-I signaling [43]. We demonstrate here that MAP3K13 phosphorylates TRIM25 at $\operatorname{Ser}_{12}$ and stabilizes it by reducing its auto-polyubiquitination.

TRIM25 belongs to the TRIM E3 ligase family. Like many other members of this family, TRIM25 targets many
HCCs from TCGA. e MAP3K13 gene copy number in normal liver and tumor tissues from TCGA. f Correlation of MAP3K13 mRNA level with its DNA copy number in HCC tissues from TCGA dataset. Each point is an individual sample. r, Spearman correlation coefficient. g Kaplan-Meier Overall Survival curves of human HCC patients with low versus high MAP3K13 expression, based on TCGA data. h TRIM25 mRNA levels in normal liver and HCCs from TCGA. i Kaplan-Meier Overall Survival curves of human HCC patients with low versus high MAP3K13 expression, based on TCGA data

proteins for ubiquitin-mediated degradation, which plays vital roles in the control of cancer cell behavior [21, 23]. In line with its function as an E3 ligase, we have shown that TRIM25 interacts with and regulates FBXW7 $\alpha$ Lys $_{48^{-}}$ linked polyubiquitination, thereby stabilization of Myc and likely promoting HCC development. TRIM25 is highly expressed in HCC, correlates with Myc expression and is associated with poor outcomes. In addition, a Myc ubiquitination assay demonstrated that TRIM25 $\mathrm{Ser}_{12}$ phosphorylation is essential for the oncogenic effect of MAP3K13. Thus, the MAP3K13-TRIM25-FBXW7 $\alpha$-Myc signaling axis is critical for promoting HCC development.

Although no other major E3 ubiquitin ligases of Myc were identified in MAP3K13 co-immunoprecipitation proteins, whether MAP3K13 regulates other such E3 ubiquitin ligase(s) of Myc remains unclear. In summary, our work indicates that MAP3K13 promotes proliferation in $\mathrm{HCC}$, by functioning as a novel post-translational regulator of Myc. 
Our findings provide further understanding of HCC development and raise the possibility that inhibiting the MAP3K13-TRIM25 axis could be an effective treatment modality for $\mathrm{HCC}$ and other tumor types in which Myc is similarly regulated.

\section{Materials and methods}

\section{Cell culture and transfection}

Human HepG2, FHCC98, and HEK293T cells were obtained from the China Center for Type Culture Collection (Wuhan, China) and were cultured as previously described [26]. For transfection, HepG2, FHCC98, and HEK293T cells were transfected using Lipofectamine 2000 (Invitrogen, Carlsbad, CA), according to the manufacturer's instructions.

\section{Plasmids and reagents}

Proteasome inhibitor MG132 (M8699) and cycloheximide (R750107) were purchased from Sigma-Aldrich. Anti-HA and anti-FLAG affinity agarose beads were purchased from Selleck (Houston, USA). All antibodies used in this study are provided in Table S3. The human MAP3K13, TRIM25, FBXW7 $\alpha$, and Myc coding sequences were amplified from HEK293T cDNA and cloned into pCMV-HA and pHAGECMV-MCS-PGK vectors. The Luc-Myc vector was generated by cloning the human Myc coding sequences to the luciferase gene tail of pcDNA3.1(+)-5'flag Luc. Mutations in the Myc, TRIM25, FBXW7 $\alpha$, and Ubiquitin cDNAs were generated by overlap extension PCR. Deletion mutants from MAP3K13 and FBXW7 $\alpha$ were cloned into pHAGECMV-MCS-PGK vectors, and the TRIM25 deletion mutants were inserted into pCMV-HA vectors. For recombinant proteins, WT TRIM25, TRIM25 S12A mutant, and MAP3K13 were cloned into the pGEX-4T-AB1 vector. Human MAP3K13 shRNAs were obtained from GENECHEM (Shanghai, China). Human TRIM25 and FBXW7 $\alpha$ shRNAs were designed and synthesized by GENEWIZ (Souzhou, China), subsequently annealed and inserted into the pLKO.1-puro vector. Human MAP3K13 and TRIM25 sgRNAs were designed by an online tool and cloned into the Lenti-CRISPER v2 vector. All primers for construction are available in Table S4.

\section{Human HCC specimens}

Human HCC samples were used as previously described [44]. Written-informed consent was obtained from patients at Union Hospital in Wuhan, China. The diagnosis of HCCs was verified by histological review.

\section{Subcutaneous xenograft}

All animal studies were approved by the Animal Care Committee of Wuhan University. Four-week-old female BALB/c nude mice were purchased from Beijing Vital River Laboratory Animal Technology (Beijing, China) and maintained in microisolator cages. For xenograft experiments, $5 \times 10^{6}$ cells were suspended in $100 \mu \mathrm{L}$ PBS and injected subcutaneously in the flanks of animals ( $n=3$ per group). Tumor growth was monitored every three days for a total period of 30 days. Tumor volumes were calculated by the equation $\mathrm{V}\left(\mathrm{mm}^{3}\right)=$ $\mathrm{a} \times \mathrm{b} \times \mathrm{c} / 2$, where $\mathrm{a}$ is the length, $\mathrm{b}$ is the width, and $\mathrm{c}$ is the height. Tumors were harvested for protein assays [26].

\section{In vitro phosphorylation assay}

Recombinant proteins of GST-TRIM25 or GST-TRIM25 S12A and GST-MAP3K13 were prepared and purified from Rossita. In a typical phosphorylation reaction, $1 \mu \mathrm{g}$ GSTTRIM25 or GST-TRIM25 S12A protein was incubated with GST-MAP3K13 in a $50 \mu \mathrm{l}$ kinase reaction buffer $(50 \mathrm{mM}$ Tris- $\mathrm{HCl}(\mathrm{pH} 7.5), 5 \mathrm{mM} \mathrm{MgCl} 2,30 \mu \mathrm{M}$ ATP) at $37^{\circ} \mathrm{C}$ for $0.5 \mathrm{~h}$. Reactions were stopped by the addition of SDS sample buffer, and samples were then heated for $5 \mathrm{~min}$ at $95^{\circ} \mathrm{C}$. Phosphorylation of TRIM25 was analysed by immuno-blot with TRIM25 and p-Ser antibodies.

\section{Luciferase reporter assays}

The Myc, FBXW7 $\alpha$, FBXL14, SKP2, and GSK3 $\beta$ cDNA fragments were amplified by PCR and cloned into the luciferase gene tail of pcDNA3.1(+)-5'flag Luc. Luciferase assays were performed as previously described [44].

\section{Lentivirus infection}

All lentiviral vectors were packaged using the manufacturer's procedures. Cells were infected with lentiviruses in the presence of polybrene. After infection, cells were selected $1 \mu \mathrm{g} / \mathrm{ml}$ puromycin for 2 weeks to obtain stable clones. The levels of protein expression in stable clones were validated by western blot.

\section{MTT assay}

For MTT assays, cells were seeded into 96-well plates $\left(10^{3}\right.$ cells/well) and cultured in DMEM supplemented with $10 \%$ FBS. The next day, fresh medium containing MTT was added and cells were incubated for $5 \mathrm{~h}$ at $37^{\circ} \mathrm{C}$. At the indicated time, this was replaced with $200 \mu$ solution of DMSO. MTT uptake was assessed in triplicate wells for each point by measuring the light absorbance at $570 \mathrm{~nm}$ using the ELX800 absorbance microplate reader. 


\section{Mass spectrometry analysis}

HEK293T cells previously transfected with the FlagMAP3K13 plasmid were lysed and immunoprecipitated with anti-Flag antibody conjugated to agarose. After SDSPAGE and Coomassie Blue staining of the immuno-precipitate, bands of interest were excised, subjected to in-gel trypsin digestion and dried. The composition of protein was analyzed by mass spectrometry according to the protocols described previously [45].

\section{Protein half-life assay}

After achieving $~ 70 \%$ confluence, cells in 12-well plates were transfected with the indicated plasmids using Lipofectamine 2000. $36 \mathrm{~h}$ later, the cells were treated with cycloheximide (CHX, $50 \mu \mathrm{g} / \mathrm{ml}$ ) for the indicated times before collection.

\section{Co-IP and immuno-blot analysis}

For Co-IP assays, HEK293T cells transfected with the indicated plasmids were lysed in $1 \mathrm{~mL}$ lysis buffer $(50 \mathrm{mM}$ Tris- $\mathrm{HCl}, \mathrm{pH} 7.4,150 \mathrm{mM} \mathrm{NaCl}, 1 \mathrm{mM}$ EDTA, $5 \mathrm{mM}$ EGTA, $1 \%$ Triton, $10 \mathrm{mg} / \mathrm{ml}$ aprotinin, $10 \mathrm{mg} / \mathrm{ml}$ leupeptin, and $1 \mathrm{mM}$ phenylmethylsulfonyl fluoride). For immunoprecipitation, the gels were washed with $0.8 \mathrm{ml}$ lysis buffer two times, then $0.9 \mathrm{ml}$ of cell lysate was added into the indicated gels and incubated overnight at $4{ }^{\circ} \mathrm{C}$. The next day, the gels were centrifuged at $3000 \mathrm{rpm}$ for $3 \mathrm{~min}$, and the supernatant was discarded. Subsequently the beads were washed with $0.8 \mathrm{ml}$ lysis buffer three times and mixed with in $2 \times \operatorname{SDS}$ sample buffer. Lysate samples were boiled for $10 \mathrm{~min}$ and were analyzed by immuno-blot with the indicated antibodies.

\section{In vivo ubiquitination assay}

Thirty-six hour after transfection, cells were treated with $20 \mu \mathrm{M}$ MG132 for $6 \mathrm{~h}$. They were then lysed in RIPA lysis buffer $(50 \mathrm{mM}$ Tris- $\mathrm{HCl}, \mathrm{pH} 7.4,150 \mathrm{mM} \mathrm{NaCl}, 1 \mathrm{mM}$ EDTA, $5 \mathrm{mM}$ EGTA, $1 \%$ Nonidet P-40, $0.1 \%$ sodium deoxycholate, $10 \mu \mathrm{g} / \mathrm{mL}$ aprotinin, $10 \mu \mathrm{g} / \mathrm{mL}$ leupeptin, and $1 \mathrm{mM}$ phenylmethylsulfonyl fluoride) and denatured by heating at $95{ }^{\circ} \mathrm{C}$ for $5 \mathrm{~min}$. Immuno-precipitation analysis was performed as described above. The samples were boiled for $10 \mathrm{~min}$ in SDS-PAGE sample buffer and analyzed by immuno-blot with the indicated antibodies.

\section{RT-qPCR}

Total RNA was isolated from cells using Trizol Reagent (Invitrogen) followed by treatment with DNase I (Thermo Scientific) and quantified using a NanoDrop 2000 spectrophotometer (NanoDrop Technologies). Subsequent reverse transcription was performed using a cDNA Synthesis Kit (Promega), according to the manufacturer's instructions. qPCR was then performed with SYBR Green Supermix (Bio-Rad, Hercules, CA) using standard procedures. All primer sequences used are listed in Table S4. $\beta$-actin was used as an internal control.

\section{Bioinformatic analysis and gene-set enrichment analysis (GSEA)}

HCC datasets were downloaded from The Cancer Genome Atlas (TCGA) data portal (http://www.tcga-data.nci.nih. gov). MAP3K13, TRIM25, and FBXW7 $\alpha$ mRNA levels were analyzed from TCGA and NCBI GEO databases (https://www.ncbi.nlm.nih.gov). For TCGA data, 10 of 374 HCC patients were excluded because of the absence of follow-up data. Finally, 364 HCC patients subjected to mRNA expression in our analysis. Kaplan-Meier curves were generated from GEPIA website (http://gepia.cancerpku.cn/index.html). Gene-set enrichment analysis (GSEA) [46] was used to obtain gene lists based on Myc targets showed significant association with MAP3K13 or TRIM25 expression in HCC cases. The probes (226556_at and 100125859_TGI_at) of MAP3K13 were used in GSE102083 and GSE25097, respectively, and the probes (224806_at and 100133918_TGI_at) of TRIM25 were used in GSE102083 and GSE25097, respectively.

\section{Statistical analysis}

For data analysis, the GraphPad Software (Version 5.01, San Diego, CA, USA) and Microsoft Excel (Excel in Microsoft Office 2013 for Windows, Microsoft Corporation, Redmond, WA, USA) were used. Statistical significance $(p<0.05)$ was performed using the two-tailed Student's $t$-test. Data are presented as the mean \pm SD or S.E.M.

Acknowledgements This work was supported by grants from the National Key R\&D Program of China (2016YFC1302300) and National Nature Science Foundation of China (81772609, 81802782).

Author contributions QZ and YL designed the study, analyzed and interpreted the data and wrote the manuscript. QZ, XL, CL, KC, MW, and YL performed most of the experiments. QZ,YL, and EP wrote the manuscript.

\section{Compliance with ethical standards}

Conflict of interest The authors declare that they have no conflict of interest.

Publisher's note: Springer Nature remains neutral with regard to jurisdictional claims in published maps and institutional affiliations. 


\section{References}

1. Mehta A, Herrera H, Block T. Glycosylation and liver cancer. Adv Cancer Res. 2015;126:257-9.

2. Torre LA, Bray F, Siegel RL, Ferlay J, Lortet-Tieulent J, Jemal A. Global cancer statistics, 2012. CA Cancer J Clin. 2015;65:87-108.

3. El-Serag HB. Hepatocellular carcinoma. $N$ Engl J Med. 2011;365:1118-27.

4. Forner A, Llovet JM, Bruix J. Hepatocellular carcinoma. Lancet. 2012;379:1245-55.

5. Villanueva A, Hernandez-Gea V, Llovet JM. Medical therapies for hepatocellular carcinoma: a critical view of the evidence. Nat Rev Gastroenterol Hepatol. 2013;10:34-42.

6. Thorgeirsson SS, Grisham JW. Molecular pathogenesis of human hepatocellular carcinoma. Nat Genet. 2002;31:339-46.

7. Liu P, Ge M, Hu J, Li X, Che L, Sun K, et al. A functional mammalian target of rapamycin complex 1 signaling is indispensable for c-Myc-driven hepatocarcinogenesis. Hepatology. 2017;66:167-81.

8. Shachaf CM, Kopelman AM, Arvanitis C, Karlsson A, Beer S, Mandl S, et al. MYC inactivation uncovers pluripotent differentiation and tumour dormancy in hepatocellular cancer. Nature. 2004;431:1112-7.

9. Dang CV, O’Donnell KA, Zeller KI, Nguyen T, Osthus RC, Li F. The c-Myc target gene network. Semin Cancer Biol. 2006;16:253-64.

10. Grim JE, Gustafson MP, Hirata RK, Hagar AC, Swanger J, Welcker M, et al. Isoform- and cell cycle-dependent substrate degradation by the Fbw7 ubiquitin ligase. J Cell Biol. 2008;181:913-20.

11. Welcker M, Orian A, Jin J, Grim JE, Harper JW, Eisenman RN, et al. The Fbw7 tumor suppressor regulates glycogen synthase kinase 3 phosphorylation-dependent c-Myc protein degradation. Proc Natl Acad Sci USA. 2004;101:9085-90.

12. Yada M, Hatakeyama S, Kamura T, Nishiyama M, Tsunematsu R, Imaki $\mathrm{H}$, et al. Phosphorylation-dependent degradation of c-Myc is mediated by the F-box protein Fbw7. EMBO J. 2004;23:2116-25.

13. Spruck CH, Strohmaier H, Sangfelt O, Muller HM, Hubalek M, Muller-Holzner E, et al. hCDC4 gene mutations in endometrial cancer. Cancer Res. 2002;62:4535-9.

14. Welcker M, Orian A, Grim JE, Eisenman RN, Clurman BE. A nucleolar isoform of the Fbw7 ubiquitin ligase regulates c-Myc and cell size. Curr Biol. 2004;14:1852-7.

15. Masaki M, Ikeda A, Shiraki E, Oka S, Kawasaki T. Mixed lineage kinase LZK and antioxidant protein-1 activate NF-kappaB synergistically. Eur J Biochem. 2003;270:76-83.

16. Ikeda A, Hasegawa $\mathrm{K}$, Masaki M, Moriguchi T, Nishida E, Kozutsumi Y, et al. Mixed lineage kinase LZK forms a functional signaling complex with JIP-1, a scaffold protein of the c-Jun NH (2)-terminal kinase pathway. J Biochem. 2001;130:773-81.

17. Sakuma H, Ikeda A, Oka S, Kozutsumi Y, Zanetta JP, Kawasaki T. Molecular cloning and functional expression of a cDNA encoding a new member of mixed lineage protein kinase from human brain. J Biol Chem. 1997;272:28622-9.

18. Edwards ZC, Trotter EW, Torres-Ayuso P, Chapman P, Wood HM, Nyswaner K, et al. Survival of head and neck cancer cells relies upon LZK kinase-mediated stabilization of mutant p53. Cancer Res. 2017;77:4961-72.

19. Jing HZ, Qiu F, Chen SZ, Su L, Qu C. [Tripartite-motif protein 25 and pyruvate kinase M2 protein expression in non-small cell lung cancer]. Nan Fang Yi Ke Da Xue Xue Bao. 2015;35:437-41.

20. Qin Y, Cui H, Zhang H. Overexpression of TRIM25 in lung cancer regulates tumor cell progression. Technol Cancer Res Treat. 2016;15:707-15.
21. Zhu Z, Wang Y, Zhang C, Yu S, Zhu Q, Hou K, et al. TRIM25 blockade by RNA interference inhibited migration and invasion of gastric cancer cells through TGF-beta signaling. Sci Rep. 2016;6:19070.

22. Sakuma M, Akahira J, Suzuki T, Inoue S, Ito K, Moriya T, et al. Expression of estrogen-responsive finger protein (Efp) is associated with advanced disease in human epithelial ovarian cancer. Gynecol Oncol. 2005;99:664-70.

23. Sun N, Xue Y, Dai T, Li X, Zheng N. Tripartite motif containing 25 promotes proliferation and invasion of colorectal cancer cells through TGF-beta signaling. Biosci Rep. 2017. https://doi.org/10. 1042/BSR20170805.

24. Suzuki T, Urano T, Tsukui T, Horie-Inoue K, Moriya T, Ishida T, et al. Estrogen-responsive finger protein as a new potential biomarker for breast cancer. Clin Cancer Res. 2005;11:6148-54.

25. Li YH, Zhong M, Zang HL, Tian XF. Mechanism of TRIM25 mediated ubiquitination of metastasis associated protein (MTA) 1 in normal liver cells. Exp Cell Res. 2018;371:250-4.

26. Han H, Chen Y, Cheng L, Prochownik EV, Li Y. microRNA-206 impairs c-Myc-driven cancer in a synthetic lethal manner by directly inhibiting MAP3K13. Oncotarget. 2016;7:16409-19.

27. Kim SY, Herbst A, Tworkowski KA, Salghetti SE, Tansey WP. Skp2 regulates Myc protein stability and activity. Mol Cell. 2003;11:1177-88.

28. von der Lehr N, Johansson S, Wu S, Bahram F, Castell A, Cetinkaya C, et al. The F-box protein Skp2 participates in c-Myc proteosomal degradation and acts as a cofactor for c-Mycregulated transcription. Mol Cell. 2003;11:1189-200.

29. Fang X, Zhou W, Wu Q. Deubiquitinase USP13 maintains glioblastoma stem cells by antagonizing FBXL14-mediated Myc ubiquitination. J Exp Med. 2017;214:245-67.

30. Gupta S, Yla-Anttila P. Herpesvirus deconjugases inhibit the IFN response by promoting TRIM25 autoubiquitination and functional inactivation of the RIG-I signalosome. PLoS Pathog. 2018;14: e1006852.

31. Meroni G, Diez-Roux G. TRIM/RBCC, a novel class of 'single protein RING finger' E3 ubiquitin ligases. Bioessays. 2005;27:1147-57.

32. Hershko A, Ciechanover A. The ubiquitin system for protein degradation. Annu Rev Biochem. 1992;61:761-807.

33. Hershko A, Ciechanover A, Varshavsky A. Basic Medical Research Award. The ubiquitin system. Nat Med. 2000;6:1073-81.

34. Min SH, Lau AW, Lee TH, Inuzuka H, Wei S, Huang P, et al. Negative regulation of the stability and tumor suppressor function of Fbw7 by the Pin1 prolyl isomerase. Mol Cell. 2012;46:771-83.

35. Welcker M, Larimore EA, Swanger J, Bengoechea-Alonso MT, Grim JE, Ericsson J, et al. Fbw7 dimerization determines the specificity and robustness of substrate degradation. Genes Dev. 2013;27:2531-6.

36. Akhoondi S, Sun D, von der Lehr N, Apostolidou S, Klotz K, Maljukova A, et al. FBXW7/hCDC4 is a general tumor suppressor in human cancer. Cancer Res. 2007;67:9006-12.

37. Coleman WB. Mechanisms of human hepatocarcinogenesis. Curr Mol Med. 2003;3:573-88.

38. Fletcher S, Prochownik EV. Small-molecule inhibitors of the Myc oncoprotein. Biochim Biophys Acta. 2015;1849:525-43.

39. Chen J, Shin JH, Zhao R, Phan L, Wang H, Xue Y, et al. CSN6 drives carcinogenesis by positively regulating Myc stability. Nat Commun. 2014;5:5384.

40. Hassan QN 2nd, Alinari L, Byrd JC. PLK1: a promising and previously unexplored target in double-hit lymphoma. J Clin Invest. 2018;128:5206-8.

41. Horiuchi D, Camarda R, Zhou AY, Yau C, Momcilovic O, Balakrishnan S, et al. PIM1 kinase inhibition as a targeted therapy 
against triple-negative breast tumors with elevated MYC expression. Nat Med. 2016;22:1321-9.

42. Gack MU, Shin YC, Joo CH, Urano T, Liang C, Sun L, et al. TRIM25 RING-finger E3 ubiquitin ligase is essential for RIG-Imediated antiviral activity. Nature. 2007;446:916-20.

43. Lee NR, Choi JY, Yoon IH, Lee JK, Inn KS. Positive regulatory role of c-Src-mediated TRIM25 tyrosine phosphorylation on RIGI ubiquitination and RIG-I-mediated antiviral signaling pathway. Cell Immunol. 2018;332:94-100.
44. Han H, Sun D, Li W, Shen H, Zhu Y, Li C, et al. A c-MycMicroRNA functional feedback loop affects hepatocarcinogenesis. Hepatology. 2013;57:2378-89.

45. Shevchenko A, Wilm M, Vorm O, Mann M. Mass spectrometric sequencing of proteins silver-stained polyacrylamide gels. Anal Chem. 1996;68:850-8.

46. Subramanian A, Kuehn H, Gould J, Tamayo P, Mesirov JP. GSEA-P: a desktop application for Gene Set Enrichment Analysis. Bioinformatics. 2007;23:3251-3. 